\title{
Six novel susceptibility loci for coronary artery disease and cerebral infarction identified by longitudinal exome-wide association studies in a Japanese population
}

\author{
YOSHIKI YASUKOCHI $^{1,2}$, JUN SAKUMA ${ }^{2-4}$, ICHIRO TAKEUCHI ${ }^{2,4,5}$, KIMIHIKO KATO $^{1,6}$, \\ MITSUTOSHI OGURI $^{1,7}$, TETSUO FUJIMAKI ${ }^{8}$, HIDEKI HORIBE $^{9}$ and YOSHIJI YAMADA $^{1,2}$
}

\begin{abstract}
${ }^{1}$ Department of Human Functional Genomics, Advanced Science Research Promotion Center, Mie University, Tsu, Mie 514-8507; ${ }^{2}$ Core Research for Evolutional Science and Technology, Japan Science and Technology Agency, Kawaguchi, Saitama 332-0012; ${ }^{3}$ Computer Science Department, College of Information Science, University of Tsukuba, Tsukuba, Ibaraki 305-8573; ${ }^{4}$ RIKEN Center for Advanced Intelligence Project, Tokyo 103-0027; ${ }^{5}$ Department of Computer Science, Nagoya Institute of Technology, Nagoya, Aichi 466-8555; ${ }^{6}$ Department of Internal Medicine, Meitoh Hospital, Nagoya, Aichi 465-0025; ${ }^{7}$ Department of Cardiology, Kasugai Municipal Hospital, Kasugai, Aichi 486-8510; ${ }^{8}$ Department of Cardiovascular Medicine, Inabe General Hospital, Inabe, Mie 511-0428; ${ }^{9}$ Department of Cardiovascular Medicine, Gifu Prefectural Tajimi Hospital, Tajimi, Gifu 507-8522, Japan
\end{abstract}

Received January 26, 2018; Accepted May 31, 2018

DOI: $10.3892 /$ br.2018.1109

\begin{abstract}
Coronary artery disease (CAD) and cerebral infarction $(\mathrm{CI})$ remain major causes of morbidity and mortality in humans. Recent genome-wide association studies have identified various genetic variants associated with these diseases. However, these studies were commonly conducted in a cross-sectional manner. Therefore, the present research performed longitudinal exome-wide association studies for CAD and CI using data on 244,000 genotyped variants and the clinical data of 6,026 Japanese individuals who had attended annual health checkups for several years (mean followed-up period, $5 \pm 3$ years). Following quality controls, the significance [false discovery rate (FDR) of <0.05] of association of the diseases with 24,651 single nucleotide polymorphisms (SNPs) in 5,989 individuals for three inheritance models was tested using the generalized estimating equation model. SNPs that reached statistical significance were further screened against a threshold of approxdf (a scale of small effective sample size) of $>30$. The longitudinal exome-wide association studies revealed that three SNPs [rs4606855 of ADGRE3 (P=2.5x10-6;
\end{abstract}

Correspondence to: Dr Yoshiki Yasukochi, Department of Human Functional Genomics, Advanced Science Research Promotion Center, Mie University, 1577 Kurima-machiya, Tsu, Mie 514-8507, Japan

E-mail: hyasukou@proof.ocn.ne.jp

Key words: cerebral infarction, coronary artery disease, exome-wide association study, generalized estimating equation, longitudinal data, single nucleotide polymorphism
FDR=0.031; approxdf=71), rs3746414 of ZFP64 $\left(\mathrm{P}=5.9 \times 10^{-6}\right.$; $\mathrm{FDR}=0.048$; approxdf $=93$ ) and $\mathrm{rs7132908}$ of FAIM2 $\left(\mathrm{P}<2.0 \times 10^{-16} ; \mathrm{FDR}<4.9 \times 10^{-12}\right.$; approxdf=65)] were significantly associated with the prevalence of CAD. A different set of three SNPs [rs6580741 of FAM186A (P<2.0x10 ${ }^{-16}$; FDR $<4.9 \times 10^{-12}$; approxdf=48), rs1324015 of LINC00400 ( $\mathrm{P}<2.0 \times 10^{-16}$; FDR $<4.9 \times 10^{-12}$; approxdf $=49$ ) and $\mathrm{rs} 884205$ of TNFRSF11A $\left(\mathrm{P}<2.0 \times 10^{-16} ; \mathrm{FDR}<4.9 \times 10^{-12}\right.$; approxdf=32)] was significantly associated with $\mathrm{CI}$. The comparison of disease incidence with these SNPs demonstrated that all the minor alleles were associated with decreased susceptibility to CAD or CI. In conclusion, six novel SNPs were identified as susceptibility loci for CAD (rs4606855 of ADGRE3, rs3746414 of ZFP64, and rs7132908 of FAIM2) or CI (rs6580741 of FAM186A, rs1324015 of LINC00400, and rs884205 of TNFRSF11A).

\section{Introduction}

Coronary artery disease (CAD) remains the worldwide leading cause of mortality among men and women (1). The American Heart Association reported that in 2014 there were 364,593 mortalities from CAD in the United States (2), based on 2014 mortality data from the National Center for Health Statistics (3). In Japan, the Ministry of Health, Labour and Welfare has reported that the rate of mortality per 100,000 population from acute ischemic heart diseases was 71,673 in 2015 (4). Cerebral infarction (CI) is also a serious clinical problem worldwide. In the United States, approximately 610,000 and 185,000 people experience new and recurrent stroke events each year, respectively, and it was estimated that the prevalence of silent CI ranged from 6-28\% from 1993 to 2005 (2). In 2015, the number of mortalities from CI and intracerebral hemorrhage in Japanese patients was 64,523 and 32,113 , respectively (4). Therefore, examination of CAD and 
CI susceptibility variants in Japanese individuals may be key to successful personalized prevention of these diseases.

Previous genome-wide association studies (GWASs) have identified a variety of genes and loci that confer susceptibility to CAD and CI across various ethnic groups, and have reported inter-ethnic differences of genetic contribution to these diseases (5-11). A meta-analysis using datasets of the CARDIoGRAMplusC4D, MIGen and CARDIoGRAM Exome consortia and ESP EOMI studies assessed the association of genetic variants in several chemokine receptor genes with CAD and myocardial infarction (6). The study did not identify any evidence of an association between the genetic variants and disease in large European ancestry cohorts, whereas six low frequency variants were associated with myocardial infarction in a South Asian cohort. Another meta-analysis of two independent Chinese GWASs for CAD identified four CAD-associated loci that were not present in populations of European ancestry (7). Inter-ethnic difference of disease susceptibility loci has also been reported in GWASs for ischemic stroke (i.e., CI). A GWAS for ischemic stroke in 6,341 Japanese individuals who participated in three independent population-based studies identified that cadherin EGF LAG seven-pass G-type receptor 1 (CELSR 1) was a susceptibility gene for ischemic stroke (10). The association of CELSR1 with stroke has been confirmed in a Portuguese cohort (12); however, the Siblings With Ischemic Stroke Study did not demonstrate an association of 312 probands with ischemic stroke from 70 centers in the USA and Canada $(13,14)$. The discrepancy of disease-susceptible variants among populations may be due to differences of genetic background among ethnic groups.

Although recent GWASs have identified a large number of genetic variants that confer susceptibility to cardiovascular diseases $(5,7,9,14,15)$, they have been conducted in a cross-sectional manner that commonly measures traits at a single point in time. To address this issue, the present study examined disease progression and physiological changes in 5,989 Japanese individuals who had annual health check-ups for several years, and performed longitudinal exome-wide association studies (EWASs) to investigate novel susceptibility loci for CAD and CI.

\section{Materials and methods}

Study subjects. The physiological data of 6,026 community-dwelling individuals recruited to a population-based cohort study (Inabe Health and Longevity Study) in Inabe City, Japan was traced (16-20). These individuals visited Inabe General Hospital for an annual health check-up, with a mean annual follow-up period of $5 \pm 3$ years (covering April 2003 to March 2014). All participants had undergone 1 to 11 medical examinations. This cohort was denoted as the 'Inabe cohort' in the longitudinal EWASs. Detailed methods for the recruitment of subjects and for the collection and storage of medical examination data and genomic DNA samples have been described previously (19). Diagnostic criteria for CAD and CI have also been described previously (18).

The study protocol complied with the Declaration of Helsinki and was approved by the Committees on the Ethics of Human Research of Mie University Graduate School of
Medicine and Inabe General Hospital (Inabe, Japan). Written informed consent was obtained from all subjects prior to enrollment in the Inabe Health and Longevity Study.

Longitudinal EWAS. Infinium HumanExome-12 ver. 1.2 BeadChip and Infinium Exome-24 ver. 1.0 BeadChip (Illumina, Inc., San Diego, CA, USA) were used to genotype $\sim 244,000$ genetic variants in the Inabe cohort for longitudinal EWASs. These arrays include putative functional exonic variants selected from $>12,000$ individual exome and whole-genome sequences across diverse ethnic populations, including European, African, Chinese and Hispanic individuals (21). Missing genotype or phenotype data of each individual was eliminated from the analyses. Quality control of genotyping data was performed, and monomorphic sites and the following genetic variants were discarded: i) Variants contained in only one of the exome arrays used ( $3.6 \%$ of all variants); ii) variants with a call rate of $<97.0 \%$; iii) variants with a minor allele frequency (MAF) of $<0.05$; iv) variants whose genotype distribution significantly deviated from Hardy-Weinberg equilibrium $(\mathrm{P}<0.001)$ in controls; and v) variants located on mitochondrial DNA or sex chromosomes.

In addition, sex specification was checked for each sample, with samples for which the sex designation in the clinical records was inconsistent with genetic sex discarded. Cryptic relatedness and duplicate samples were checked by calculation of identity by descent (IBD); all pairs of DNA samples exhibiting an IBD of $>0.1875$ were inspected and one sample from each pair was excluded. Population stratification in the Inabe cohort was examined by principal component analysis via the EIGENSTRAT method (22), using JMP Genomics version 6.0 (SAS Institute, Inc., Cary, NC, USA), and four outliers were removed from the longitudinal EWASs. Consequently, a total of 24,651 single nucleotide polymorphisms (SNPs) among 5,989 Japanese individuals passed quality control for the longitudinal EWASs of CAD and CI. The rearrangement of Inabe longitudinal data was conducted using R software version 3.32 (23) via RStudio version 1.0.136 (http://www.rstudio.com/) (24) and Perl script (version 5.26.2; https://www.perl.org/get.html). Using JMP Genomics, genotyping data were converted into numeric data for dominant, additive and recessive inheritance models. The dominant and recessive models were defined as ' $0, \mathrm{AA} ; 1$, $\mathrm{AB}+\mathrm{BB}$ ' and '0, $\mathrm{AA}+\mathrm{AB}$; 1 , BB' (A, major allele; $\mathrm{B}$, minor allele), respectively, whereas the additive model was defined as ' $0, \mathrm{AA} ; 1, \mathrm{AB} ; 2$, BB'.

Statistical analysis. Longitudinal EWASs were conducted for 5,713 control individuals, 170 subjects with CAD, and 117 with CI (Table I). The association of the prevalence of CAD and CI with SNPs was tested by the generalized estimating equation (GEE) model $(25,26)$ with adjustments for age, sex, body mass index (BMI), smoking and prevalence of hypertension, type 2 diabetes mellitus and dyslipidemia, using the R package 'geepack' (27). Since the prevalence of CAD and CI is repeated categorical data (case or control), a binomial distribution was applied for assessing the association between the categorical outcomes and SNPs in the GEE method. The waves argument was used 
Table I. Longitudinal characteristics of study subjects in the Inabe cohort.

\begin{tabular}{|c|c|c|c|}
\hline Characteristic & Control $^{\mathrm{a}}$ & $\mathrm{CAD}^{\mathrm{a}}$ & $\mathrm{CI}^{\mathrm{a}}$ \\
\hline No. of subjects ${ }^{b}$ & 5,713 & 170 & 117 \\
\hline Sex, male/female, $\%^{\mathrm{b}}$ & $54.7 / 45.3$ & $77.1 / 22.9$ & $68.4 / 31.6$ \\
\hline Age, years & $52.0 \pm 0.07(27,143)$ & $63.1 \pm 0.41(619)$ & $63.4 \pm 0.35(466)$ \\
\hline Body mass index, $\mathrm{kg} / \mathrm{m}^{2}$ & $22.9 \pm 0.02(26,719)$ & $23.9 \pm 0.13(507)$ & $23.6 \pm 0.16(416)$ \\
\hline Current or former smoker, $\%$ & $38.4(27,143)$ & $40.1(619)$ & $35.0(466)$ \\
\hline Hypertension, \% & $31.9(26,715)$ & $75.7(503)$ & $65.1(415)$ \\
\hline Systolic blood pressure, $\mathrm{mmHg}$ & $120.4 \pm 0.10(26,715)$ & $126.5 \pm 0.77(503)$ & $127.6 \pm 0.81(415)$ \\
\hline Diastolic blood pressure, $\mathrm{mmHg}$ & $74.6 \pm 0.08(26,715)$ & $76.5 \pm 0.55(503)$ & $77.5 \pm 0.58(415)$ \\
\hline Type 2 diabetes mellitus, $\%$ & $11.4(26,745)$ & $38.8(590)$ & $36.4(464)$ \\
\hline Fasting plasma glucose, $\mathrm{mmol} / \mathrm{l}$ & $5.59 \pm 0.007(26,731)$ & $6.49 \pm 0.086(586)$ & $6.22 \pm 0.098(464)$ \\
\hline Blood hemoglobin $\mathrm{A}_{1 \mathrm{c}}, \%$ & $5.69 \pm 0.004(19,870)$ & $6.18 \pm 0.052(465)$ & $6.03 \pm 0.041(410)$ \\
\hline Dyslipidemia, $\%$ & $58.4(26,713)$ & $76.5(592)$ & $77.9(444)$ \\
\hline Serum triglycerides, $\mathrm{mmol} / \mathrm{l}$ & $1.25 \pm 0.006(26,710)$ & $1.48 \pm 0.040(591)$ & $1.36 \pm 0.037(444)$ \\
\hline Serum HDL-cholesterol, mmol/1 & $1.61 \pm 0.003(26,687)$ & $1.43 \pm 0.019(585)$ & $1.51 \pm 0.020(440)$ \\
\hline Serum LDL-cholesterol, mmol/1 & $3.19 \pm 0.005(25,594)$ & $2.76 \pm 0.032(563)$ & $3.20 \pm 0.039(422)$ \\
\hline Chronic kidney disease, $\%$ & $10.8(24,461)$ & $37.6(582)$ & $37.0(441)$ \\
\hline Serum creatinine, $\mu \mathrm{mol} / \mathrm{l}$ & $74.1 \pm 0.58(24,461)$ & $132.7 \pm 8.65(582)$ & $163.0 \pm 13.33(441)$ \\
\hline 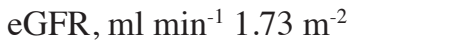 & $79.2 \pm 0.11(24,461)$ & $66.1 \pm 1.11(582)$ & $64.5 \pm 1.31(441)$ \\
\hline Hyperuricemia, \% & $18.1(23,985)$ & $42.3(575)$ & $27.5(436)$ \\
\hline Serum uric acid, $\mu \mathrm{mol} / 1$ & $327.7 \pm 0.56(23,985)$ & $367.3 \pm 4.20(575)$ & $352.7 \pm 4.09(436)$ \\
\hline
\end{tabular}

${ }^{a}$ Values in parentheses indicate the number of measurements taken; ${ }^{b}$ numbers based on examination data from the final visit for each subject. Quantitative data are presented as the mean and standard error of the mean. CAD, coronary artery disease; CI, cerebral infarction; HDL, high-density lipoprotein; LDL, low-density lipoprotein; eGFR, estimated glomerular filtration rate.

to specify the ordering of repeated measurements within individuals. Effects of SNPs in exome arrays on CAD or CI are not independent as many SNPs are in linkage disequilibrium (LD) $(13,28,29)$. Therefore, the false discovery rate (FDR) was calculated using the Benjamin and Hochberg method (30) to compensate for multiple comparison of genotypes with the phenotypes. An FDR of $<0.05$ was considered to indicate a statistically significant association.

A small effective sample size may increase the probability of generating false positives (type I errors) (31). Sitlani et al (31) recommend the use of approxdf, which is a scale of small effective sample size: Approxdf $=2$ x MAF x Nindep, where Nindep is the sum of the estimated number of independent observations per person. They demonstrated that an approxdf of $\geq 10$ could reduce type I errors. Thus, approxdf was computed by the R package 'bosswithdf' $(31,32)$. To avoid the issue of false positive association in small sample sizes, a strict approxdf threshold was applied, and SNPs with approxdf $\leq 30$ discarded.

LD estimates and prediction of functional association for candidate loci. To survey LDs between the candidate SNPs detected in the present study and previously identified CADor CI-associated SNPs in JPT (Japanese in Tokyo, Japan) from the 1000 Genomes Project [http://www.1000genomes. org/ (33)], analyses were conducted using the LDproxy of LDlink [https://analysistools.nci.nih.gov/LDlink/ (34)], which is a web-based application. The association of SNPs with CAD or CI reported by previous studies was investi- gated using the Genome-Wide Repository of Associations Between SNPs and Phenotypes [GRASP; https://grasp. nhlbi.nih.gov/Overview.aspx (35)], DisGeNET [http://www. disgenet.org/web/DisGeNET/ (36)] and GWAS Catalogue [https://www.ebi.ac.uk/gwas/ (37)] databases. Gene-gene functional interactions were predicted using the GeneMANIA Cytoscape plugin (38-40) via Cytoscape version 3.4.0 software [http://www.cytoscape.org/ (41)].

\section{Results}

Characteristics of subjects. The characteristics of subjects in the Inabe cohort with respect to longitudinal data are presented in Table I. The prevalence of hypertension, type 2 diabetes mellitus, dyslipidemia, chronic kidney disease and hyperuricemia was higher in patients with CAD or CI than in controls. The prevalence of CAD and CI was lower in females (39 CAD and $37 \mathrm{CI}$ patients) than in males (131 CAD and 80 CI patients). The majority of physiological or clinical parameters examined (age, BMI, systolic and diastolic blood pressure, fasting plasma glucose level, blood hemoglobin $A_{1 c}$ content and serum concentrations of triglycerides, creatinine, and uric acid) were higher, whereas serum concentrations of high-density lipoprotein-cholesterol and estimated glomerular filtration rate were lower, in the patients than in the controls. The serum concentration of low-density lipoprotein-cholesterol was lower in patients with CAD $(2.76 \pm 0.032 \mathrm{mmol} / \mathrm{l})$ than in controls $(3.19 \pm 0.005 \mathrm{mmol} / \mathrm{l})$. This discrepancy may be 
attributable to effects of lipid-lowering treatment for patients with CAD.

Association of SNPs with longitudinal data on the prevalence of $C A D$. Following the quality control checks, the association between 24,651 SNPs and the prevalence of CAD in the Inabe cohort was tested by using the GEE model with adjustments for age, sex, BMI, smoking and prevalence of hypertension, type 2 diabetes mellitus and dyslipidemia. Candidate SNPs that reached statistical significance (FDR of $<0.05)$ were additionally screened against the threshold of approxdf $(>30)$. Analyses revealed that two SNPs [rs4606855 of ADGRE3 $\left(\mathrm{P}=2.5 \times 10^{-6} ; \mathrm{FDR}=0.031\right.$, approxdf $\left.=71\right)$ and $\mathrm{rs} 3746414$ of ZFP64 ( $\mathrm{P}=5.9 \times 10^{-6}$; FDR=0.048; approxdf $\left.\left.=93\right)\right]$ and one SNP [rs7132908 of FAIM2 $\left(\mathrm{P}<2.0 \times 10^{-16}\right.$; FDR $<4.9 \times 10^{-12}$; approxdf $=65$ )] were significantly associated with the prevalence of CAD in additive and recessive models, respectively (Table II).

According to the GRASP, DisGeNET and GWAS Catalogue databases, the association of the three SNPs with CAD has not been reported to date. Therefore, the three SNPs were identified as novel genetic variants that may confer susceptibility to CAD. The nucleotide substitution at rs7132908 in FAIM2 is predicted to be a silent substitution in the 3'-untranslated region, while the remaining two SNPs are predicted to alter amino acid residues (i.e., nonsynonymous substitutions), according to the NCBI dbSNP database [https://www.ncbi. nlm.nih.gov/projects/SNP/ (42)].

Genotype distributions for rs4606855, rs3746414 and rs7132908 in subjects with CAD and controls in the longitudinal EWAS are listed in Table III. In the current study, the prevalence of CAD was lower in subjects with minor alleles than in those with major alleles for all SNPs, suggesting the CC genotype of rs 4606855 and the AA genotypes of rs3746414 and rs7132908 to be protective against CAD.

Association of SNPs with longitudinal data on the prevalence of CI. In the Inabe cohort, three SNPs [rs6580741 of FAM186A $\left(\mathrm{P}<2.0 \times 10^{-16} ; \mathrm{FDR}<4.9 \times 10^{-12}\right.$; approxdf $\left.=48\right)$, rs1324015 of LINC00400 ( $\mathrm{P}<2.0 \times 10^{-16}$; FDR $<4.9 \times 10^{-12}$; approxdf=49), and $\mathrm{rs} 884205$ of TNFRSF11A $\left(\mathrm{P}<2.0 \times 10^{-16}\right.$; FDR $<4.9 \times 10^{-12}$; approxdf $=32$ )] were significantly associated with the prevalence of CI in the recessive model, although the GEE tests in the dominant and additive models detected no significant association between SNPs and the prevalence of CI (Table II). The CI-associated SNPs detected in the recessive model were not shared with the CAD-associated SNPs. The nucleotide substitution at rs6580741 in FAM186A is predicted to alter an amino acid residue (Table II), whereas the substitutions at the other SNPs are predicted as silent, according to the NCBI dbSNP.

The rs6580741 of FAM186A is located relatively close $(\sim 464 \mathrm{~kb})$ to rs7132908 of FAIM2 at chromosomal region 12q13.12, although these SNPs were related to the different diseases. The LD among these SNPs was thus examined among participants in the Inabe cohort using the R package 'genetics' [https://CRAN.R-project.org/package=genetics (43)]. The estimation demonstrated that these SNPs were not in LD $\left(D^{\prime}=0.045, r^{2}=0.002\right)$, suggesting that rs7132908 and rs6580741 were independently associated with the prevalence of CAD and CI, respectively. 
Table III. Genotype distributions for three candidate SNPs among subjects with CAD and controls.

\begin{tabular}{lccccc}
\hline RefSNP ID & Position $^{\mathrm{a}}$ & Gene & Genotype & Controls $^{\mathrm{b}}$ & CAD $^{\mathrm{b}}$ \\
\hline rs4606855 & $19: 14,658,527$ & ADGRE3 & GG & $21,095(77.7)$ & $564(91.1)$ \\
& & & GC & $5,676(20.9)$ & $54(8.7)$ \\
rs3746414 & \multirow{2}{*}{$20: 52,152,840$} & ZFP64 & CC & $372(1.4)$ & $1(0.2)$ \\
& & & GG & $20,122(74.1)$ & $529(85.5)$ \\
& & GA & $6,506(24.0)$ & $81(13.1)$ \\
rs7132908 & FAIM2 & AA & $515(1.9)$ & $9(1.5)$ \\
& $12: 49,869,365$ & GG & $13,609(50.1)$ & $344(55.6)$ \\
& & GA & $11,128(41.0)$ & $252(40.7)$ \\
& & AA & $2,406(8.9)$ & $23(3.7)$ \\
\hline
\end{tabular}

${ }^{a}$ Position in NCBI Genome Reference Consortium Human Build 38; ${ }^{b}$ values indicate the numbers of measurements taken, with the percentages in parentheses. SNP, single nucleotide polymorphism; CAD, coronary artery disease.

Table IV. Genotype distributions for three candidate SNPs among subjects with CI and controls.

\begin{tabular}{lccccc}
\hline RefSNP ID & Position $^{\mathrm{a}}$ & Gene & Genotype & Controls $^{\mathrm{b}}$ & CI $^{\mathrm{b}}$ \\
\hline rs6580741 & 12: $50,333,923$ & FAM186A & GG & $14,669(54.1)$ & $269(57.7)$ \\
& & GC & $10,555(38.9)$ & $171(36.7)$ \\
& & CC & $1,900(7.0)$ & $26(5.6)$ \\
rs1324015 & LINC00400 & GG & $14,809(54.6)$ & $295(63.3)$ \\
& & & GA & $10,433(38.5)$ & $171(36.7)$ \\
rs884205 & & AA & $1,882(6.9)$ & $0(0.0)$ \\
& TNFRSF11A & GG & $16,573(61.1)$ & $312(67.0)$ \\
& & & GT & $9,154(33.7)$ & $154(33.0)$ \\
& & & TT & $1,397(5.2)$ & $0(0.0)$ \\
\hline
\end{tabular}

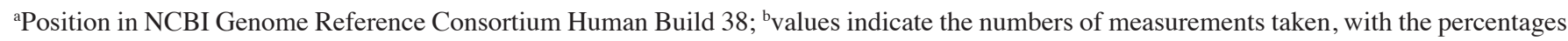
in parentheses. SNP, single nucleotide polymorphism; CI, cerebral infarction.

Genotype distributions for rs6580741, rs1324015 and rs884205 in subjects with CI and controls in the longitudinal EWAS are listed in Table IV. As with the case of CAD-associated SNPs, for all three SNPs, the prevalence of CI was lower in subjects with minor alleles than in those with major alleles. The $C C$ genotype of rs6580741, the $A A$ genotype of rs1324015, and the TT genotype of rs 884205 may thus be protective against CI.

Assessment of LD between the six identified SNPs and other SNPs associated with CAD or CI. The LDs between the six candidate SNPs identified in the present study and adjacent SNPs across a $\sim 1 \mathrm{Mb}$ genomic region were assessed using the LDproxy in LDlink. The LDproxy analysis indicated that 433 SNPs were in significant $L D\left(r^{2} \geq 0.5\right)$ with one of the candidate SNPs in JPT from the 1000 Genomes Project (Table V). Of the 433 SNPs, there were no SNPs previously identified to be associated with CAD or $\mathrm{CI}(\mathrm{P}>0.01)$ according to the GRASP database. In addition, the LDpair analysis in LDlink using allele frequency data in East Asian populations from the 1000 Genomes Project indicated that rs1324015 associated with the prevalence of $\mathrm{CI}$ in the present study was not in LD with rs9533425 $\left(\mathrm{D}^{\prime}=0.4709, \mathrm{r}^{2}=0.0007\right)$, previously reported to be associated with lag time (lag phase of the turbidimetric clotting assay) to fibrin clot formation (28). These results suggest that effects of the candidate SNPs on the prevalence of CAD or CI are independent.

Gene interaction network analysis. To investigate the interactive functional association, a GeneMANIA network analysis was conducted of the top ten genes (high gene-disease association scores) that have been demonstrated to be associated with CAD or CI selected from the DisGeNET database, and of the five genes (ZFP64, FAIM2, ADGRE3, FAM186A, and TNFRSF11A) identified in the present study (Fig. 1). Given that LINC00400 non-coding RNA has not been well characterized, it could not be examined. The network analysis showed that the CAD- and CI-associated genes identified in the present study have potential direct or indirect interactions with several genes previously demonstrated to be associated with CAD and CI, respectively. The network suggested that ZFP64 and FAIM2 interact with $L O X$ and both $C R P$ and $A P O B$, respectively. 
Table V. Correlation estimates of candidate (query) SNPs with adjacent SNPs using LDproxy.

\begin{tabular}{|c|c|c|c|c|c|c|c|c|}
\hline Chr. pos. & RefSNP ID & $r^{2}$ & Chr. pos. & RefSNP ID & $r^{2}$ & Chr. pos. & RefSNP ID & $r^{2}$ \\
\hline $18 \mathrm{q} 21.33$ & rs884205 & 1.00 & $20 \mathrm{q} 13.2$ & rs12481281 & 0.77 & $12 \mathrm{q} 13.12$ & rs7979830 & 1.00 \\
\hline $13 q 14.11$ & rs1324015 & 1.00 & $20 \mathrm{q} 13.2$ & rs3787176 & 0.77 & $12 \mathrm{q} 13.12$ & rs35723625 & 1.00 \\
\hline $13 q 14.11$ & rs2589313 & 0.59 & $20 \mathrm{q} 13.2$ & rs72626582 & 0.77 & $12 q 13.12$ & rs10876013 & 1.00 \\
\hline $13 q 14.11$ & rs1324016 & 0.57 & $20 \mathrm{q} 13.2$ & rs72626583 & 0.77 & $12 q 13.12$ & rs12425229 & 1.00 \\
\hline $13 q 14.11$ & rs2762188 & 0.57 & $20 \mathrm{q} 13.2$ & rs6021711 & 0.76 & $12 \mathrm{q} 13.12$ & rs3812825 & 1.00 \\
\hline $13 q 14.11$ & rs2589314 & 0.57 & $20 \mathrm{q} 13.2$ & rs2180366 & 0.76 & $12 q 13.12$ & rs1362983 & 1.00 \\
\hline $13 q 14.11$ & rs35574382 & 0.57 & $20 \mathrm{q} 13.2$ & rs67904269 & 0.76 & $12 q 13.12$ & rs 141978158 & 1.00 \\
\hline $13 q 14.11$ & rs2762185 & 0.57 & $20 q 13.2$ & rs7273288 & 0.74 & $12 \mathrm{q} 13.12$ & rs7308095 & 1.00 \\
\hline $13 q 14.11$ & rs2657098 & 0.57 & $20 \mathrm{q} 13.2$ & rs13045200 & 0.61 & $12 q 13.12$ & rs 11169319 & 1.00 \\
\hline $13 q 14.11$ & rs 1562123 & 0.57 & $20 \mathrm{q} 13.2$ & rs11478756 & 0.61 & $12 q 13.12$ & rs12811291 & 1.00 \\
\hline $13 q 14.11$ & rs 11424017 & 0.52 & $20 \mathrm{q} 13.2$ & rs11473560 & 0.56 & $12 q 13.12$ & rs34825838 & 1.00 \\
\hline $20 q 13.2$ & rs3746414 & 1.00 & $20 \mathrm{q} 13.2$ & rs6021739 & 0.56 & $12 \mathrm{q} 13.12$ & rs11169317 & 1.00 \\
\hline $20 q 13.2$ & rs3746413 & 1.00 & $20 \mathrm{q} 13.2$ & rs6096795 & 0.56 & $12 q 13.12$ & rs3861100 & 1.00 \\
\hline 20q13.2 & rs3746415 & 1.00 & $20 \mathrm{q} 13.2$ & rs6021738 & 0.56 & $12 q 13.12$ & rs 11169315 & 1.00 \\
\hline 20q13.2 & rs3787181 & 1.00 & $20 \mathrm{q} 13.2$ & rs6021737 & 0.56 & $12 \mathrm{q} 13.12$ & rs2009072 & 1.00 \\
\hline $20 q 13.2$ & rs 11431529 & 1.00 & $20 \mathrm{q} 13.2$ & rs6021736 & 0.56 & $12 \mathrm{q} 13.12$ & rs 11169314 & 1.00 \\
\hline $20 \mathrm{q} 13.2$ & rs67642347 & 1.00 & $20 \mathrm{q} 13.2$ & rs6013400 & 0.56 & $12 \mathrm{q} 13.12$ & rs6580739 & 0.97 \\
\hline $20 q 13.2$ & rs4809893 & 1.00 & $20 \mathrm{q} 13.2$ & rs6096794 & 0.56 & $12 \mathrm{q} 13.12$ & rs7972824 & 0.97 \\
\hline $20 q 13.2$ & rs8122116 & 1.00 & $20 \mathrm{q} 13.2$ & rs6013399 & 0.56 & $12 \mathrm{q} 13.12$ & rs9739363 & 0.97 \\
\hline $20 \mathrm{q} 13.2$ & rs6021732 & 1.00 & $20 \mathrm{q} 13.2$ & rs117265881 & 0.56 & $12 \mathrm{q} 13.12$ & rs12823506 & 0.97 \\
\hline $20 q 13.2$ & rs2224227 & 1.00 & $20 \mathrm{q} 13.2$ & rs4809892 & 0.51 & $12 q 13.12$ & rs112456855 & 0.97 \\
\hline $20 q 13.2$ & rs66953446 & 1.00 & $20 \mathrm{q} 13.2$ & rs 145753021 & 0.51 & $12 q 13.12$ & rs12422417 & 0.97 \\
\hline $20 q 13.2$ & rs115523244 & 1.00 & 19p13.12 & rs4606855 & 1.00 & $12 q 13.12$ & rs35224873 & 0.97 \\
\hline $20 \mathrm{q} 13.2$ & rs6021723 & 1.00 & $19 \mathrm{p} 13.12$ & rs56819877 & 1.00 & $12 \mathrm{q} 13.12$ & rs12828340 & 0.87 \\
\hline $20 q 13.2$ & rs12480406 & 1.00 & $19 \mathrm{p} 13.12$ & rs4808971 & 1.00 & $12 \mathrm{q} 13.12$ & rs17124562 & 0.87 \\
\hline $20 q 13.2$ & rs67638808 & 1.00 & $19 \mathrm{p} 13.12$ & rs7255012 & 1.00 & $12 \mathrm{q} 13.12$ & rs11169335 & 0.87 \\
\hline $20 q 13.2$ & rs67262858 & 1.00 & $19 p 13.12$ & rs10426121 & 1.00 & $12 q 13.12$ & rs35576436 & 0.87 \\
\hline 20q13.2 & rs6013397 & 1.00 & 19p13.12 & rs6511956 & 1.00 & $12 q 13.12$ & rs 11169332 & 0.87 \\
\hline $20 q 13.2$ & rs12481222 & 0.97 & $19 p 13.12$ & rs4273164 & 1.00 & $12 q 13.12$ & rs 11169331 & 0.87 \\
\hline $20 \mathrm{q} 13.2$ & rs139218250 & 0.97 & $19 \mathrm{p} 13.12$ & rs4239642 & 1.00 & $12 \mathrm{q} 13.12$ & rs71465002 & 0.87 \\
\hline $20 q 13.2$ & rs7346642 & 0.97 & $19 p 13.12$ & rs10402993 & 0.95 & $12 q 13.12$ & rs7314465 & 0.87 \\
\hline $20 q 13.2$ & rs67892028 & 0.97 & $19 \mathrm{p} 13.12$ & rs11085902 & 0.95 & $12 \mathrm{q} 13.12$ & rs6580730 & 0.87 \\
\hline $20 q 13.2$ & rs73130324 & 0.97 & $19 p 13.12$ & rs11085901 & 0.95 & $12 q 13.12$ & rs7308885 & 0.87 \\
\hline $20 q 13.2$ & rs75085690 & 0.97 & $19 p 13.12$ & rs7245656 & 0.86 & $12 q 13.12$ & rs2358539 & 0.87 \\
\hline $20 q 13.2$ & rs3838014 & 0.94 & $19 p 13.12$ & rs7250114 & 0.86 & $12 q 13.12$ & rs 201343445 & 0.87 \\
\hline $20 q 13.2$ & rs3179313 & 0.94 & $19 p 13.12$ & rs73506161 & 0.86 & $12 q 13.12$ & rs12424713 & 0.87 \\
\hline $20 \mathrm{q} 13.2$ & rs3179314 & 0.94 & $19 \mathrm{p} 13.12$ & rs35805282 & 0.86 & $12 \mathrm{q} 13.12$ & rs12424691 & 0.87 \\
\hline $20 q 13.2$ & rs3787178 & 0.94 & 19 p13.12 & rs75540045 & 0.86 & $12 \mathrm{q} 13.12$ & rs11169322 & 0.87 \\
\hline $20 q 13.2$ & rs 1555328 & 0.94 & 19 p13.12 & rs34930135 & 0.86 & $12 \mathrm{q} 13.12$ & rs12425705 & 0.87 \\
\hline $20 \mathrm{q} 13.2$ & rs6021748 & 0.94 & $19 \mathrm{p} 13.12$ & rs373306807 & 0.66 & $12 \mathrm{q} 13.12$ & rs35628283 & 0.87 \\
\hline 20q13.2 & rs6021747 & 0.94 & $12 q 13.12$ & rs6580741 & 1.00 & $12 q 13.12$ & rs35875720 & 0.87 \\
\hline $20 q 13.2$ & rs4811297 & 0.94 & $12 q 13.12$ & rs7310541 & 1.00 & $12 q 13.12$ & rs34198664 & 0.84 \\
\hline $20 \mathrm{q} 13.2$ & rs111897744 & 0.94 & $12 q 13.12$ & rs7134337 & 1.00 & $12 \mathrm{q} 13.12$ & rs11292692 & 0.82 \\
\hline $20 q 13.2$ & rs6021745 & 0.94 & $12 q 13.12$ & rs7134595 & 1.00 & $12 \mathrm{q} 13.12$ & rs4768905 & 0.77 \\
\hline $20 q 13.2$ & rs6021744 & 0.94 & $12 q 13.12$ & rs55931113 & 1.00 & $12 \mathrm{q} 13.12$ & rs59210472 & 0.77 \\
\hline $20 q 13.2$ & rs6021743 & 0.94 & $12 q 13.12$ & rs35878271 & 1.00 & $12 \mathrm{q} 13.12$ & rs4636745 & 0.77 \\
\hline $20 q 13.2$ & rs6021742 & 0.94 & $12 q 13.12$ & rs34039674 & 1.00 & $12 q 13.12$ & rs11830586 & 0.77 \\
\hline $20 \mathrm{q} 13.2$ & rs6021741 & 0.94 & $12 q 13.12$ & rs66895907 & 1.00 & $12 q 13.12$ & rs7973910 & 0.77 \\
\hline $20 \mathrm{q} 13.2$ & rs6021740 & 0.94 & $12 q 13.12$ & rs4768900 & 1.00 & $12 \mathrm{q} 13.12$ & rs73305103 & 0.77 \\
\hline $20 \mathrm{q} 13.2$ & rs6021735 & 0.94 & $12 q 13.12$ & rs10876020 & 1.00 & $12 \mathrm{q} 13.12$ & rs4321029 & 0.77 \\
\hline 20q13.2 & rs6021734 & 0.94 & $12 q 13.12$ & rs34858415 & 1.00 & $12 q 13.12$ & rs7968898 & 0.77 \\
\hline $20 \mathrm{q} 13.2$ & rs56783695 & 0.94 & $12 q 13.12$ & $\mathrm{rs} 11836169$ & 1.00 & $12 \mathrm{q} 13.12$ & rs 151015253 & 0.77 \\
\hline
\end{tabular}


Table V. Continued.

\begin{tabular}{|c|c|c|c|c|c|c|c|c|}
\hline Chr. pos. & RefSNP ID & $\mathrm{r}^{2}$ & Chr. pos. & RefSNP ID & $\mathrm{r}^{2}$ & Chr. pos. & RefSNP ID & $\mathrm{r}^{2}$ \\
\hline $20 q 13.2$ & rs60610902 & 0.94 & $12 q 13.12$ & rs4768949 & 1.00 & $12 \mathrm{q} 13.12$ & rs11169323 & 0.74 \\
\hline $20 q 13.2$ & rs6021733 & 0.94 & $12 q 13.12$ & rs4768872 & 1.00 & $12 q 13.12$ & rs73305105 & 0.74 \\
\hline 20q13.2 & rs60614585 & 0.94 & $12 q 13.12$ & rs34614542 & 1.00 & $12 q 13.12$ & rs35925338 & 0.74 \\
\hline 20q13.2 & rs57913961 & 0.94 & $12 q 13.12$ & rs12312177 & 1.00 & $12 \mathrm{q} 13.12$ & NA & 0.72 \\
\hline $20 q 13.2$ & rs6021730 & 0.94 & $12 q 13.12$ & rs34894919 & 1.00 & $12 \mathrm{q} 13.12$ & rs 144615146 & 0.72 \\
\hline $20 q 13.2$ & rs6021729 & 0.94 & $12 q 13.12$ & rs12424876 & 1.00 & $12 q 13.12$ & rs78601155 & 0.72 \\
\hline 20q13.2 & rs6021728 & 0.94 & $12 q 13.12$ & rs11169377 & 1.00 & $12 \mathrm{q} 13.12$ & rs74336127 & 0.72 \\
\hline $20 q 13.2$ & rs6021727 & 0.94 & $12 q 13.12$ & rs11169376 & 1.00 & $12 q 13.12$ & rs141852922 & 0.69 \\
\hline 20q13.2 & rs6096791 & 0.94 & $12 q 13.12$ & rs36017775 & 1.00 & $12 \mathrm{q} 13.12$ & rs 145504356 & 0.69 \\
\hline 20q13.2 & rs6091458 & 0.94 & $12 q 13.12$ & rs11169375 & 1.00 & $12 \mathrm{q} 13.12$ & rs34098872 & 0.65 \\
\hline $20 q 13.2$ & rs6091457 & 0.94 & $12 q 13.12$ & rs7137319 & 1.00 & $12 q 13.12$ & rs67576611 & 0.64 \\
\hline $20 q 13.2$ & rs200887094 & 0.94 & $12 q 13.12$ & rs7295847 & 1.00 & $12 q 13.12$ & rs7138622 & 0.64 \\
\hline 20q13.2 & rs6096784 & 0.94 & $12 q 13.12$ & rs7296291 & 1.00 & $12 q 13.12$ & rs7138420 & 0.64 \\
\hline $20 q 13.2$ & rs77290230 & 0.94 & $12 q 13.12$ & rs7312252 & 1.00 & $12 q 13.12$ & rs7315690 & 0.64 \\
\hline $20 q 13.2$ & rs12624632 & 0.94 & $12 q 13.12$ & rs10506292 & 1.00 & $12 q 13.12$ & rs5798135 & 0.63 \\
\hline 20q13.2 & rs6021722 & 0.94 & $12 q 13.12$ & rs34849043 & 1.00 & $12 \mathrm{q} 13.12$ & rs17124514 & 0.62 \\
\hline $20 q 13.2$ & rs6091453 & 0.94 & $12 q 13.12$ & rs10615610 & 1.00 & $12 q 13.12$ & rs12424335 & 0.62 \\
\hline $20 q 13.2$ & rs6021721 & 0.94 & $12 q 13.12$ & rs10876023 & 1.00 & $12 q 13.12$ & rs34145380 & 0.62 \\
\hline $20 q 13.2$ & rs6021720 & 0.94 & $12 q 13.12$ & rs10876024 & 1.00 & $12 q 13.12$ & rs34309034 & 0.62 \\
\hline $20 q 13.2$ & rs6512796 & 0.94 & $12 q 13.12$ & rs7301186 & 1.00 & $12 q 13.12$ & rs34245511 & 0.62 \\
\hline 20q13.2 & rs12480343 & 0.93 & $12 q 13.12$ & rs11169374 & 1.00 & $12 \mathrm{q} 13.12$ & rs9364 & 0.62 \\
\hline $20 q 13.2$ & rs12480321 & 0.93 & $12 q 13.12$ & rs11169373 & 1.00 & $12 q 13.12$ & rs8181651 & 0.60 \\
\hline $20 q 13.2$ & rs67451131 & 0.93 & $12 q 13.12$ & rs35209607 & 1.00 & $12 q 13.12$ & rs80003859 & 0.60 \\
\hline $20 q 13.2$ & rs6013393 & 0.93 & $12 q 13.12$ & rs4421818 & 1.00 & $12 q 13.12$ & rs144939089 & 0.60 \\
\hline $20 q 13.2$ & rs67862732 & 0.93 & $12 q 13.12$ & rs11169370 & 1.00 & $12 q 13.12$ & rs28364704 & 0.59 \\
\hline $20 q 13.2$ & rs 144698812 & 0.93 & $12 q 13.12$ & rs11169369 & 1.00 & $12 q 13.12$ & rs736167 & 0.59 \\
\hline $20 q 13.2$ & rs117427009 & 0.93 & $12 q 13.12$ & rs 11169390 & 1.00 & $12 q 13.12$ & rs10783347 & 0.59 \\
\hline $20 q 13.2$ & rs 140670817 & 0.93 & $12 q 13.12$ & rs11169391 & 1.00 & $12 q 13.12$ & rs10783346 & 0.59 \\
\hline $20 \mathrm{q} 13.2$ & rs6021709 & 0.93 & $12 q 13.12$ & rs4445717 & 1.00 & $12 q 13.12$ & rs61928279 & 0.59 \\
\hline $20 q 13.2$ & rs6021708 & 0.93 & $12 q 13.12$ & rs9668187 & 1.00 & $12 q 13.12$ & rs7302422 & 0.59 \\
\hline $20 q 13.2$ & rs72626578 & 0.93 & $12 q 13.12$ & rs60025018 & 1.00 & $12 q 13.12$ & rs12423130 & 0.59 \\
\hline 20q13.2 & rs6021707 & 0.93 & $12 q 13.12$ & rs11169393 & 1.00 & $12 \mathrm{q} 13.12$ & rs3815671 & 0.59 \\
\hline $20 q 13.2$ & rs6021706 & 0.93 & $12 q 13.12$ & rs10467106 & 1.00 & $12 q 13.12$ & rs57583527 & 0.59 \\
\hline $20 q 13.2$ & rs 140980517 & 0.93 & $12 q 13.12$ & rs12303082 & 1.00 & $12 q 13.12$ & rs10747572 & 0.59 \\
\hline $20 q 13.2$ & rs 143481833 & 0.93 & $12 q 13.12$ & rs7971374 & 1.00 & $12 q 13.12$ & rs71083515 & 0.57 \\
\hline $20 q 13.2$ & rs12624823 & 0.93 & $12 q 13.12$ & rs10783352 & 1.00 & $12 q 13.12$ & rs79043170 & 0.55 \\
\hline $20 q 13.2$ & rs12624819 & 0.93 & $12 q 13.12$ & rs10783353 & 1.00 & $12 q 13.12$ & rs35404088 & 0.55 \\
\hline $20 q 13.2$ & rs 148218979 & 0.93 & $12 q 13.12$ & rs12299758 & 1.00 & $12 q 13.12$ & rs10783340 & 0.55 \\
\hline $20 q 13.2$ & rs6021760 & 0.90 & $12 q 13.12$ & rs12299669 & 1.00 & $12 q 13.12$ & rs7978904 & 0.55 \\
\hline $20 \mathrm{q} 13.2$ & rs3818198 & 0.90 & $12 q 13.12$ & rs11169394 & 1.00 & $12 q 13.12$ & rs10783338 & 0.55 \\
\hline $20 \mathrm{q} 13.2$ & rs72626580 & 0.90 & $12 q 13.12$ & rs11169395 & 1.00 & $12 q 13.12$ & rs7398567 & 0.55 \\
\hline $20 q 13.2$ & rs4811301 & 0.90 & $12 q 13.12$ & rs11169367 & 1.00 & $12 q 13.12$ & rs10735824 & 0.55 \\
\hline $20 q 13.2$ & rs4811303 & 0.90 & $12 q 13.12$ & rs11833608 & 1.00 & $12 q 13.12$ & rs7972465 & 0.55 \\
\hline $20 q 13.2$ & rs4811304 & 0.90 & $12 q 13.12$ & rs113486728 & 1.00 & $12 q 13.12$ & rs6580728 & 0.55 \\
\hline $20 q 13.2$ & rs4811305 & 0.90 & $12 q 13.12$ & rs376666931 & 1.00 & $12 q 13.12$ & rs7138945 & 0.55 \\
\hline $20 q 13.2$ & rs 140407501 & 0.90 & $12 q 13.12$ & rs7980911 & 1.00 & $12 q 13.12$ & rs11169282 & 0.55 \\
\hline $20 q 13.2$ & rs6021763 & 0.90 & $12 q 13.12$ & rs7302363 & 1.00 & $12 q 13.12$ & rs57061317 & 0.53 \\
\hline $20 q 13.2$ & rs6021764 & 0.90 & $12 q 13.12$ & rs4768855 & 1.00 & $12 q 13.12$ & rs76382737 & 0.53 \\
\hline $20 q 13.2$ & rs6021765 & 0.90 & $12 q 13.12$ & rs6580737 & 1.00 & $12 q 13.12$ & rs12369104 & 0.53 \\
\hline $20 q 13.2$ & rs6021766 & 0.90 & $12 q 13.12$ & rs7488682 & 1.00 & $12 q 13.12$ & rs12819883 & 0.53 \\
\hline $20 q 13.2$ & rs 11480360 & 0.90 & $12 q 13.12$ & rs7972068 & 1.00 & $12 q 13.12$ & rs3741562 & 0.53 \\
\hline 20q13.2 & rs6021767 & 0.90 & $12 q 13.12$ & rs7972202 & 1.00 & $12 q 13.12$ & rs113411336 & 0.53 \\
\hline
\end{tabular}


Table V. Continued.

\begin{tabular}{|c|c|c|c|c|c|c|c|c|}
\hline Chr. pos. & RefSNP ID & $r^{2}$ & Chr. pos. & RefSNP ID & $r^{2}$ & Chr. pos. & RefSNP ID & $\mathrm{r}^{2}$ \\
\hline $20 \mathrm{q} 13.2$ & rs6021768 & 0.90 & $12 q 13.12$ & rs6580743 & 1.00 & $12 q 13.12$ & rs34632215 & 0.53 \\
\hline $20 \mathrm{q} 13.2$ & rs6013409 & 0.90 & $12 q 13.12$ & rs4348979 & 1.00 & $12 q 13.12$ & rs11169278 & 0.53 \\
\hline $20 q 13.2$ & rs6021770 & 0.90 & $12 q 13.12$ & rs 11169360 & 1.00 & $12 q 13.12$ & rs1554845 & 0.53 \\
\hline $20 q 13.2$ & rs2038429 & 0.90 & $12 q 13.12$ & rs 11169359 & 1.00 & $12 q 13.12$ & rs2204684 & 0.53 \\
\hline $20 \mathrm{q} 13.2$ & rs6021772 & 0.90 & $12 q 13.12$ & rs12814094 & 1.00 & $12 q 13.12$ & rs2204683 & 0.53 \\
\hline $20 q 13.2$ & rs 2273472 & 0.90 & $12 q 13.12$ & rs7315955 & 1.00 & $12 q 13.12$ & rs10876000 & 0.53 \\
\hline $20 q 13.2$ & rs 2038430 & 0.90 & $12 q 13.12$ & rs 11169357 & 1.00 & $12 q 13.12$ & rs7967979 & 0.53 \\
\hline $20 q 13.2$ & rs6021774 & 0.90 & $12 q 13.12$ & rs10876028 & 1.00 & $12 q 13.12$ & rs34167640 & 0.53 \\
\hline $20 q 13.2$ & rs58878184 & 0.90 & $12 q 13.12$ & rs7135777 & 1.00 & $12 q 13.12$ & rs7307469 & 0.53 \\
\hline $20 q 13.2$ & rs72626581 & 0.90 & $12 q 13.12$ & rs11838347 & 1.00 & $12 q 13.12$ & rs1554844 & 0.53 \\
\hline $20 q 13.2$ & rs34963386 & 0.90 & $12 q 13.12$ & rs12426444 & 1.00 & $12 q 13.12$ & rs7961065 & 0.53 \\
\hline $20 q 13.2$ & NA & 0.90 & $12 q 13.12$ & rs9705460 & 1.00 & $12 q 13.12$ & rs7961112 & 0.53 \\
\hline $20 \mathrm{q} 13.2$ & rs6021775 & 0.90 & $12 q 13.12$ & rs7304445 & 1.00 & $12 q 13.12$ & rs7294618 & 0.53 \\
\hline $20 q 13.2$ & rs1973951 & 0.90 & $12 q 13.12$ & rs11323536 & 1.00 & $12 q 13.12$ & rs7307230 & 0.53 \\
\hline $20 q 13.2$ & rs77286509 & 0.90 & $12 q 13.12$ & rs7311973 & 1.00 & $12 q 13.12$ & rs7135322 & 0.53 \\
\hline $20 q 13.2$ & rs 7265436 & 0.90 & $12 q 13.12$ & rs10876017 & 1.00 & $12 q 13.12$ & rs59262224 & 0.53 \\
\hline $20 q 13.2$ & rs6096760 & 0.90 & $12 q 13.12$ & rs10876016 & 1.00 & $12 q 13.12$ & rs17124432 & 0.53 \\
\hline $20 q 13.2$ & rs 142762031 & 0.87 & $12 q 13.12$ & rs7974648 & 1.00 & $12 q 13.12$ & rs12369049 & 0.53 \\
\hline $20 q 13.2$ & rs 138459074 & 0.87 & $12 q 13.12$ & rs12821454 & 1.00 & $12 q 13.12$ & rs7308474 & 0.53 \\
\hline $20 q 13.2$ & rs67243058 & 0.84 & $12 q 13.12$ & rs7305995 & 1.00 & $12 q 13.12$ & rs7968119 & 0.53 \\
\hline $20 q 13.2$ & rs6021731 & 0.82 & $12 q 13.12$ & rs35933908 & 1.00 & $12 q 13.12$ & rs61529910 & 0.51 \\
\hline $20 \mathrm{q} 13.2$ & rs6013396 & 0.79 & $12 q 13.12$ & rs12832940 & 1.00 & $12 q 13.12$ & NA & 0.50 \\
\hline $20 q 13.2$ & rs6021719 & 0.79 & $12 q 13.12$ & rs10876015 & 1.00 & $12 q 13.12$ & rs7132908 & 1.00 \\
\hline $20 q 13.2$ & rs6021718 & 0.79 & $12 q 13.12$ & rs10876014 & 1.00 & $12 q 13.12$ & rs3205718 & 1.00 \\
\hline $20 q 13.2$ & rs6091452 & 0.79 & $12 q 13.12$ & rs67138019 & 1.00 & $12 q 13.12$ & rs12146733 & 0.98 \\
\hline $20 q 13.2$ & rs6091451 & 0.79 & $12 q 13.12$ & rs11169351 & 1.00 & $12 q 13.12$ & rs1893492 & 0.98 \\
\hline $20 q 13.2$ & rs6126474 & 0.79 & $12 q 13.12$ & rs 11169350 & 1.00 & $12 q 13.12$ & rs 145103902 & 0.98 \\
\hline $20 q 13.2$ & rs6123134 & 0.79 & $12 q 13.12$ & rs 11169349 & 1.00 & $12 q 13.12$ & rs73116325 & 0.98 \\
\hline $20 q 13.2$ & rs8121769 & 0.79 & $12 q 13.12$ & rs35535298 & 1.00 & $12 q 13.12$ & rs 146875448 & 0.85 \\
\hline $20 q 13.2$ & rs6021716 & 0.79 & $12 q 13.12$ & rs2111988 & 1.00 & $12 q 13.12$ & rs11169199 & 0.80 \\
\hline $20 q 13.2$ & rs6021715 & 0.79 & $12 q 13.12$ & rs7311491 & 1.00 & $12 q 13.12$ & rs297924 & 0.80 \\
\hline $20 q 13.2$ & rs6123133 & 0.79 & $12 q 13.12$ & rs7311378 & 1.00 & $12 q 13.12$ & rs17201502 & 0.80 \\
\hline $20 q 13.2$ & rs6096776 & 0.79 & $12 q 13.12$ & rs 11169348 & 1.00 & $12 q 13.12$ & rs73116335 & 0.80 \\
\hline $20 q 13.2$ & rs62216897 & 0.79 & $12 q 13.12$ & rs6580735 & 1.00 & $12 q 13.12$ & rs73116338 & 0.80 \\
\hline $20 q 13.2$ & rs6126472 & 0.79 & $12 q 13.12$ & rs 11169347 & 1.00 & $12 q 13.12$ & rs73116339 & 0.80 \\
\hline $20 q 13.2$ & rs 7265434 & 0.79 & $12 q 13.12$ & rs 11169345 & 1.00 & $12 q 13.12$ & rs 145512623 & 0.75 \\
\hline $20 q 13.2$ & rs 7273176 & 0.79 & $12 q 13.12$ & rs7956468 & 1.00 & $12 q 13.12$ & NA & 0.59 \\
\hline $20 q 13.2$ & rs7273121 & 0.79 & $12 q 13.12$ & rs 1972611 & 1.00 & $12 q 13.12$ & rs 149596227 & 0.57 \\
\hline $20 q 13.2$ & rs8183138 & 0.79 & $12 q 13.12$ & rs10783344 & 1.00 & $12 q 13.12$ & rs373555454 & 0.55 \\
\hline $20 q 13.2$ & rs6123131 & 0.79 & $12 q 13.12$ & rs35768991 & 1.00 & $12 q 13.12$ & rs12367809 & 0.53 \\
\hline $20 q 13.2$ & rs79718879 & 0.79 & $12 q 13.12$ & rs11169339 & 1.00 & $12 q 13.12$ & rs7306275 & 0.51 \\
\hline $20 q 13.2$ & rs76931275 & 0.79 & $12 q 13.12$ & rs7486747 & 1.00 & $12 q 13.12$ & rs7138803 & 0.50 \\
\hline $20 q 13.2$ & rs6021714 & 0.79 & $12 q 13.12$ & rs7953953 & 1.00 & $12 q 13.12$ & rs112502508 & 0.50 \\
\hline $20 q 13.2$ & rs6021713 & 0.79 & $12 q 13.12$ & rs7132551 & 1.00 & & & \\
\hline
\end{tabular}

SNPs with $\mathrm{r}^{2}>0.5$ only are shown. The candidate SNPs identified in the present study are shown in bold. SNP, single nucleotide polymorphism; Chr. pos., chromosomal position; NA, not available.

In addition, $A D G R E 3$ and TNFRSF11A were indicated to be co-expressed with $A L O X 5 A P$ and $C D 4 O L G$. The network also suggested that TNFRSF11A interacts and is co-expressed with
$P F K C H$ and $A D H 1 B$, respectively. Additionally, in the network, FAM186A was indirectly connected with CI-associated genes (NPY and F5) through TMPRSS15. 


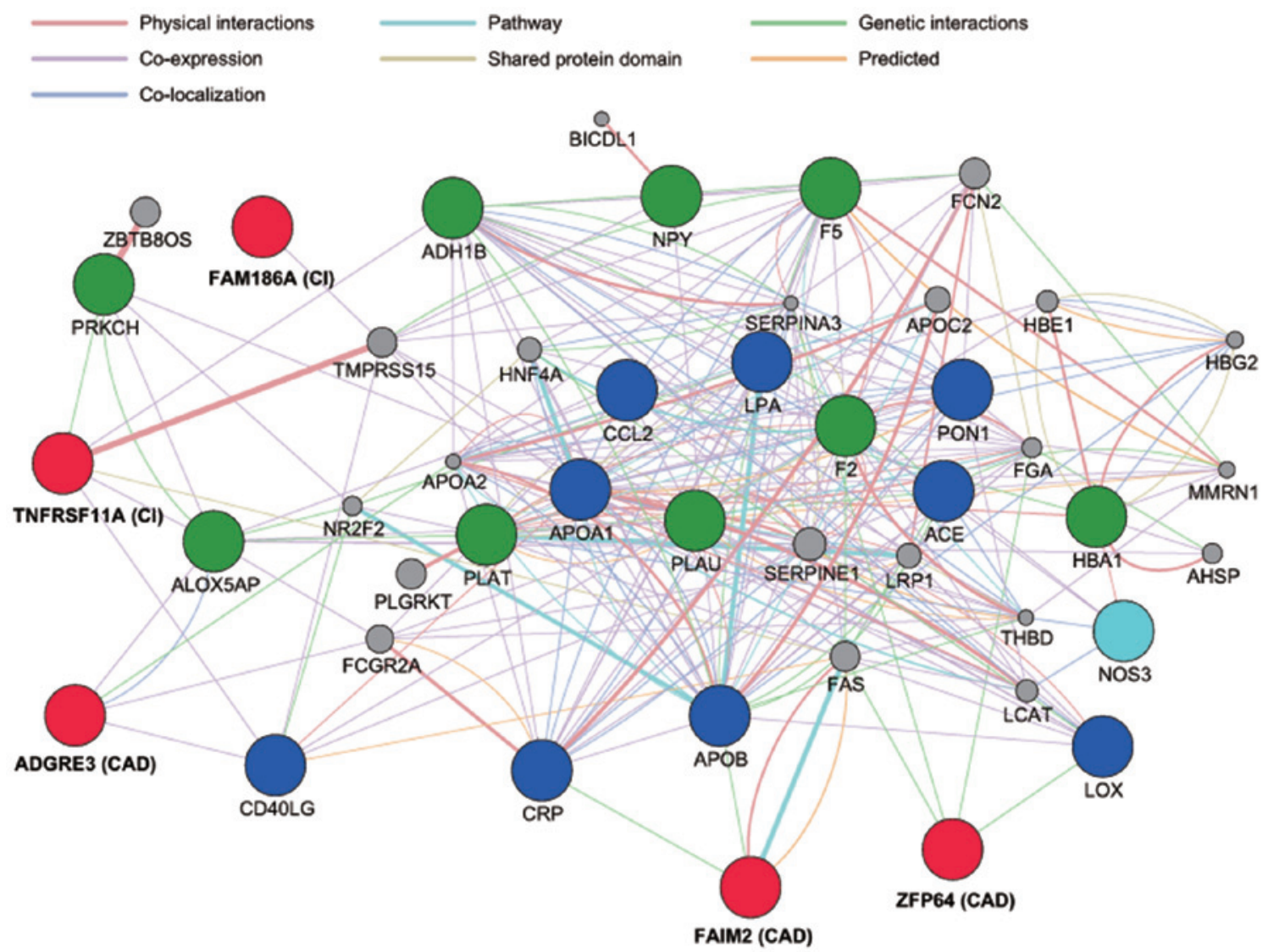

Figure 1. Diagram of GeneMANIA network for the presently identified genes (closed red circles) and previously reported genes associated with CAD (closed blue circles) and CI (closed green circles) or both (closed light blue circles). The phenotypes (CAD or CI) associated with the identified genes are shown in parentheses. CAD, coronary artery disease. CI, cerebral infarction.

\section{Discussion}

In the present study, genetic variants that confer susceptibility to CAD and CI were investigated. The longitudinal EWASs in a Japanese cohort identified that rs 4606855 of ADGRE3, rs3746414 of ZFP64 and rs7132908 of FAIM2 were significantly associated with the prevalence of CAD, whereas rs6580741 of FAM186A, rs1324015 of LINC00400 and rs884205 of TNFRSF11A were significantly associated with the prevalence of CI. According to the databases, these SNPs may be novel susceptibility loci for CAD or CI.

The adhesion $\mathrm{G}$ protein-coupled receptor E3 (ADGRE3) gene is located at chromosomal region 19p13.12, and rs4606855 $[\mathrm{G} / \mathrm{C}(\mathrm{E} 75 \mathrm{Q})]$ in this gene was significantly associated with the prevalence of CAD. The mRNA of ADGRE3 is predominantly expressed in neutrophils, monocytes and macrophages, and this protein may be important for interactions between myeloid cells including neutrophils and monocytes during immune and inflammatory responses (44). Given that vascular inflammatory responses serve a role in the development of coronary atherosclerosis and thrombosis (45-47), this CAD-associated SNP may be attributable to functional alteration of ADGRE3 protein in vascular inflammation.

The ZFP64 zinc finger protein (ZFP64) gene located at 20q13.2 is expressed in various tissues and organs, according to The Human Protein Atlas [http://www.proteinatlas. org/ $(48,49)]$. The present longitudinal EWASs demonstrated that rs3746414 [G/A $(\mathrm{S} 451 \mathrm{~N})]$ was significantly associated with the prevalence of CAD. ZFP64 protein may be a positive regulator of Toll-like receptor-induced innate immune responses through tumor necrosis factor (TNF)- $\alpha$, interleukin- 6 and interferon- $\beta$ production in macrophages (50). In fact, Zfp 64 knockdown mice exhibited significant reductions in the production of these cytokines (50). Thus, the association of ZFP64 with CAD may be attributable to the effect of this gene on the development of coronary atherosclerosis or thrombosis.

The Fas apoptotic inhibitory molecule 2 (FAIM2) gene is located at 12q13.12, and rs7132908 (G/A) in this gene was associated with the prevalence of CAD. FAIM2 is predominantly expressed in the brain while moderate expressions are also observed in other various tissues and organs, according to The Human Protein Atlas. AIM2 inhibits Fas-mediated apoptosis (51). The Fas cell surface death receptor gene $(F A S)$ serves important roles in the regulation of the immune system through inducing apoptosis of autoreactive or antigen-presenting $\mathrm{T}$ lymphocytes (52-54). Given that FAIM2 regulates Fas-mediated apoptosis during immune responses, rs7132908 in this gene may be associated with the incidence of CAD through altering the regulation of immune or inflammatory responses.

The TNF receptor superfamily member 11a (TNFRSF11A) gene is located at chromosomal region 18q21.33, and is expressed in various tissues and organs including the brain, according to The Human Protein Atlas. In the present study, rs884205 (G/T) of TNFRSF11A was significantly associated with the prevalence of CI. TNFRSF11A and its ligand 
(TNFRSF11) may be important regulators of the interaction between $T$ cells and dendritic cells that are involved in immune surveillance $(55,56)$. In mice, TNFRSF11/TNFRSF11A (also known as RANKL/RANK) signaling could trigger inflammatory fever responses in the central nervous system (55), and may serve anti-inflammatory roles in ischemic brains (56). The rs884205 in TNFRSF11A may thus influence the development of CI.

The exact functions of proteins encoded by the family with sequence similarity 186 member A (FAM186A) and long intergenic non-protein coding RNA 400 (LINC00400) genes are unknown. However, it has been reported that rs 9533425 located near LINC00400 at chromosomal region 13q14.11 demonstrated a significant association $\left(\mathrm{P}=1.9 \times 10^{-9}\right)$ with lag time to fibrin clot formation in 2,100 subjects from the Twins United Kingdom (TwinsUK) registry in stage 1 study, although the association of this SNP was not replicated in stage 2 study (28). In the present longitudinal EWASs, rs1324015 of LINC00400 was significantly associated with the prevalence of CI. According to LDpair in LDlink, rs1324015 was not in LD with rs9533425 in East Asian populations. Although the functional relevance of the candidate SNP to the development of cerebral atherosclerosis or thrombosis remains unclear, LINC00400 may be a susceptibility locus for the incidence of CI.

There were certain limitations in the present study. First, the longitudinal EWASs were conducted in only a local Japanese population, and the observed number of patients who were affected by the target diseases was not sufficient. Thus, replication of longitudinal EWASs in other Japanese populations or other ethnic groups is required to verify the association of the identified SNPs with the diseases of interest. However, to the best of our knowledge, longitudinal data for CAD and $\mathrm{CI}$ in other populations are unavailable at present. Second, no experiments for functional analyses were conducted in the present study. Thus, the functional relevance of the candidate SNPs identified by longitudinal EWASs to the pathogenesis of the diseases of interest remains unclear. Due to the lack of experiments for functional analyses, the association of the SNPs identified in the present study with CAD or CI should be interpreted with caution. Further functional analyses are required to clarify the present results.

In conclusion, rs4606855 of ADGRE3, rs3746414 of ZFP64 and rs7132908 of FAIM2 may be susceptibility loci for CAD. Additionally, the SNPs rs6580741 of FAM186A, rs1324015 of LINC00400 and rs884205 of TNFRSF11A may be genetic determinants of CI. All minor alleles of the six candidate SNPs exhibited an association with lower prevalence of the associated diseases, compared with the corresponding major alleles. This suggests that the minor alleles of each candidate SNP may be protective against CAD or CI.

\section{Acknowledgements}

Not applicable.

\section{Funding}

The present study was supported by a research grant from the Okasan Kato Culture Promotion Foundation (to Y Yasukochi), a Kurata grant awarded by the Hitachi Global Foundation (grant no. 1323 to YYasukochi and YYamada), the Core Research for Evolutional Science and Technology of the Japan Science and Technology Agency (grant no. JPMRJCR1302 to YYamada, JS and IT), and by KAKENHI grants from the Japan Society for the Promotion of Science (grant no. JP17H00758 to IT and YYasukochi; grant no. JP15H04772 to YYamada).

\section{Availability of data and materials}

The datasets used and/or analyzed during the current study are available from the corresponding author on reasonable request; if justified, applicable parts of the data can be made available in anonymized format.

\section{Authors' contributions}

YYasukochi contributed to analysis and interpretation of the data, and to drafting of the manuscript. JS and IT contributed to analysis and interpretation of the data as well as revision of the manuscript. KK, MO, TF and $\mathrm{HH}$ each contributed to acquisition of the data and revision of the manuscript. YYamada contributed to conception and design of the study, and to acquisition, analysis and interpretation of the data, and revision of the manuscript. All authors read and approved the final manuscript.

\section{Ethics approval and consent to participate}

All procedures performed in studies involving human participants were in accordance with the 1964 Helsinki declaration and its later amendments or comparable ethical standards. The study protocol was approved by the Committees on the Ethics of Human Research of Mie University Graduate School of Medicine and Inabe General Hospital (Mie, Japan). Informed consent was obtained from all individual participants included in the study.

\section{Patient consent for publication}

All participants provided written informed consent permitting publication of relevant data following anonymization of personal information.

\section{Competing interests}

The authors declare that they have no competing interests.

\section{References}

1. GBD 2013 Mortality and Causes of Death Collaborators: Global, regional, and national age-sex specific all-cause and cause-specific mortality for 240 causes of death, 1990-2013: A systematic analysis for the Global Burden of Disease Study 2013. Lancet 385: 117-171, 2015.

2. Benjamin EJ, Blaha MJ, Chiuve SE, Cushman M, Das SR, Deo R, de Ferranti SD, Floyd J, Fornage M, Gillespie C, et al; American Heart Association Statistics Committee and Stroke Statistics Subcommittee: Heart disease and stroke statistics-2017 update: A report from the American Heart Association. Circulation 135: e146-e603, 2017.

3. National Center for Health Statistics: Mortality multiple cause micro-data files, 2014: Public-use data file and documentation: NHLBI tabulations.

4. Ministry of Health, Labour and Welfare, Japan: Vital statistics. 
5. Schunkert H, König IR, Kathiresan S, Reilly MP, Assimes TL, Holm H, Preuss M, Stewart AFR, Barbalic M, Gieger C, et al; Cardiogenics; CARDIoGRAM Consortium: Large-scale association analysis identifies 13 new susceptibility loci for coronary artery disease. Nat Genet 43: 333-338, 2011.

6. Golbus JR, Stitziel NO, Zhao W, Xue C, Farrall M, McPherson R, Erdmann J, Deloukas P, Watkins H, Schunkert H, et al; CARDIoGRAMplusC4D, Myocardial Infarction Genetics (MIGen), Exome Sequencing Project and Early-Onset Myocardia Infarction (ESP EOMI), and the Pakistan Risk of Myocardial Infarction Study (PROMIS) Consortia*: Common and rare genetic variation in CCR2, CCR5, or CX3CR1 and risk of atherosclerotic coronary heart disease and glucometabolic traits. Circ Cardiovase Genet 9: 250-258, 2016.

7. Lu X, Wang L, Chen S, He L, Yang X, Shi Y, Cheng J, Zhang L, Gu CC, Huang J, et al; Coronary ARtery DIsease Genome-Wide Replication And Meta-Analysis (CARDIoGRAM) Consortium: Genome-wide association study in Han Chinese identifies four new susceptibility loci for coronary artery disease. Nat Genet 44 : 890-894, 2012.

8. Nikpay M, Goel A, Won H-H, Hall LM, Willenborg C, Kanoni S, Saleheen D, Kyriakou T, Nelson CP, Hopewell JC, et al: A comprehensive 1,000 Genomes-based genome-wide association meta-analysis of coronary artery disease. Nat Genet 47: 1121-1130, 2015.

9. Wakil SM,RamR,Muiya NP,Mehta M,AndresE,MazharN,BazB, Hagos S, Alshahid M, Meyer BF, et al: A genome-wide association study reveals susceptibility loci for myocardial infarction/coronary artery disease in Saudi Arabs. Atherosclerosis o245: 62-70, 2016.

10. Yamada Y, Fuku N, Tanaka M, Aoyagi Y, Sawabe M, Metoki N Yoshida H, Satoh K, Kato K, Watanabe S, et al: Identification of CELSR 1 as a susceptibility gene for ischemic stroke in Japanese individuals by a genome-wide association study. Atherosclerosis 207: 144-149, 2009

11. Kubo M,Hata J, Ninomiya T, Matsuda K, Yonemoto K, Nakano T, Matsushita T, Yamazaki K, Ohnishi Y, Saito S, et al: A nonsynonymous SNP in PRKCH (protein kinase $\mathrm{C} \eta$ ) increases the risk of cerebral infarction. Nat Genet 39: 212-217, 2007.

12. Gouveia LO, Sobral J, Vicente AM, Ferro JM and Oliveira SA: Replication of the CELSR1 association with ischemic stroke in a Portuguese case-control cohort. Atherosclerosis 217: 260-262, 2011.

13. Akinyemi RO, Ovbiagele B, Akpalu A, Jenkins C, Sagoe K Owolabi L, Sarfo F, Obiako R, Gebreziabher M, Melikam E, et al SIREN Investigators as Members of the H3Africa Consortium: Stroke genomics in people of African ancestry: Charting new paths. Cardiovasc J Afr 26 (Suppl 1): S39-S49, 2015.

14. Meschia JF, Nalls M, Matarin M, Brott TG, Brown RD Jr, Hardy J, Kissela B, Rich SS, Singleton A, Hernandez D, et al Siblings With Ischemic Stroke Study Investigators: Siblings with ischemic stroke study: Results of a genome-wide scan for stroke loci. Stroke 42: 2726-2732, 2011.

15. Kilarski LL, Achterberg S, Devan WJ, Traylor M, Malik R, Lindgren A, Pare G, Sharma P, Slowik A, Thijs V, et al; GARNET Collaborative Research Group, Wellcome Trust Case Control Consortium 2, Australian Stroke Genetic Collaborative, the METASTROKE Consortium, and the International Stroke Genetics Consortium: Meta-analysis in more than 17,900 cases of ischemic stroke reveals a novel association at $12 \mathrm{q} 24.12$. Neurology 83 . 678-685, 2014.

16. Yamada Y, Matsui K, Takeuchi I and Fujimaki T: Association of genetic variants with dyslipidemia and chronic kidney disease in a longitudinal population-based genetic epidemiological study. Int J Mol Med 35: 1290-1300, 2015.

17. Yamada Y, Matsui K, Takeuchi I, Oguri M and Fujimaki T: Association of genetic variants of the $\alpha$-kinase 1 gene with type 2 diabetes mellitus in a longitudinal population-based genetic epidemiological study. Biomed Rep 3: 347-354, 2015.

18. Yamada Y, Matsui K, Takeuchi I and Fujimaki T: Association of genetic variants with coronary artery disease and ischemic stroke in a longitudinal population-based genetic epidemiological study. Biomed Rep 3: 413-419, 2015.

19. Yamada Y, Matsui K, Takeuchi I, Oguri M and Fujimaki T: Association of genetic variants with hypertension in a longitudina population-based genetic epidemiological study. Int J Mol Med 35 : $1189-1198,2015$

20. Oguri M, Fujimaki T, Horibe H, Kato K, Matsui K, Takeuchi I and Yamada Y: Obesity-related changes in clinical parameters and conditions in a longitudinal population-based epidemiological study. Obes Res Clin Pract 11: 299-314, 2017.
21. Grove ML, Yu B, Cochran BJ, Haritunians T, Bis JC, Taylor KD, Hansen M, Borecki IB, Cupples LA, Fornage M, et al: Best practices and joint calling of the HumanExome BeadChip: The CHARGE Consortium. PLoS One 8: e68095, 2013

22. Price AL, Patterson NJ, Plenge RM, Weinblatt ME, Shadick NA and Reich D: Principal components analysis corrects for stratification in genome-wide association studies. Nat Genet 38: 904-909, 2006.

23. R Development Core Team: R: A language and environment for statistical computing. Vienna, Austria, 2016.

24. RStudio Team: RStudio: Integrated development environment for R. RStudio, Inc., Boston, MA, 2015.

25. Liang KY and Zeger SL: Longitudinal data analysis using generalized linear models. Biometrika 73: 13-22, 1986.

26. Hanley JA, Negassa A, Edwardes MD and Forrester JE: Statistical analysis of correlated data using generalized estimating equations: An orientation. Am J Epidemiol 157: 364-375, 2003.

27. Halekoh U, Højsgaard S and Yan J: The R package geepack for generalized estimating equations. J Stat Softw 15: 1-11, 2006

28. Williams FMK, Carter AM, Hysi PG, Surdulescu G, Hodgkiss D, Soranzo N, Traylor M, Bevan S, Dichgans M, Rothwell PMW, et al; EuroCLOT Investigators; Wellcome Trust Case Control Consortium 2; MOnica Risk, Genetics, Archiving and Monograph; MetaStroke; International Stroke Genetics Consortium: Ischemic stroke is associated with the ABO locus: The EuroCLOT study. Ann Neurol 73: 16-31, 2013.

29. Holmen OL, Zhang H, Fan Y, Hovelson DH, Schmidt EM, Zhou W, Guo Y, Zhang J, Langhammer A, Løchen ML, et al: Systematic evaluation of coding variation identifies a candidate causal variant in TM6SF2 influencing total cholesterol and myocardial infarction risk. Nat Genet 46: 345-351, 2014

30. Benjamini Y and Hochberg Y: Controlling the false discovery rate: A practical and powerful approach to multiple testing. J R Stat Soc B 57: 289-300, 1995.

31. Sitlani CM, Rice KM, Lumley T, McKnight B, Cupples LA, Avery CL, Noordam R, Stricker BHC, Whitsel EA and Psaty BM: Generalized estimating equations for genome-wide association studies using longitudinal phenotype data. Stat Med 34: 118-130, 2015.

32. Voorman A, Rice K and Lumley T: Fast computation for genomewide association studies using boosted one-step statistics. Bioinformatics 28: 1818-1822, 2012.

33. 1000 Genomes Project Consortium; Abecasis GR, Altshuler D, Auton A, Brooks LD, Durbin RM, Gibbs RA, Hurles ME, McVean GA: A map of human genome variation from population-scale sequencing. Nature 467: 1061-1073, 2010.

34. Machiela MJ and Chanock SJ: LDlink: A web-based application for exploring population-specific haplotype structure and linking correlated alleles of possible functional variants. Bioinformatics 31: 3555-3557, 2015.

35. Leslie R, O'Donnell CJ and Johnson AD: GRASP: Analysis of genotype-phenotype results from 1390 genome-wide association studies and corresponding open access database. Bioinformatics 30: i185-i194, 2014.

36. Piñero J, Queralt-Rosinach N, Bravo À, Deu-Pons J, BauerMehren A, Baron M, Sanz F and Furlong LI: DisGeNET: A discovery platform for the dynamical exploration of human diseases and their genes. Database (Oxford) 2015: bav028, 2015.

37. MacArthur J, Bowler E, Cerezo M, Gil L, Hall P, Hastings E, Junkins H, McMahon A, Milano A, Morales J, et al: The new NHGRI-EBI Catalog of published genome-wide association studies (GWAS Catalog). Nucleic Acids Res 45 (D1): D896-D901, 2017.

38. Warde-Farley D, Donaldson SL, Comes O, Zuberi K, Badrawi R, Chao P, Franz M, Grouios C, Kazi F, Lopes CT, et al: The GeneMANIA prediction server: Biological network integration for gene prioritization and predicting gene function. Nucleic Acids Res 38: W214-220, 2010.

39. Montojo J, Zuberi K, Rodriguez H, Bader GD and Morris Q: GeneMANIA: Fast gene network construction and function prediction for Cytoscape. F1000Res 3: 153, 2014.

40. Montojo J,Zuberi K, Rodriguez H, Kazi F, Wright G, Donaldson SL, Morris Q and Bader GD: GeneMANIA Cytoscape plugin: Fast gene function predictions on the desktop. Bioinformatics 26: 2927-2928, 2010.

41. Shannon P, Markiel A, Ozier O, Baliga NS, Wang JT, Ramage D, Amin N, Schwikowski B and Ideker T: Cytoscape: A software environment for integrated models of biomolecular interaction networks. Genome Res 13: 2498-2504, 2003. 
42. Sherry ST, Ward MH, Kholodov M, Baker J, Phan L, Smigielski EM and Sirotkin K: dbSNP: The NCBI database of genetic variation. Nucleic Acids Res 29: 308-311, 2001.

43. Warnes $G$, with contributions from Gorjanc G, Leisch F and Man M: Genetics: Population Genetics, 2013.

44. Stacey M, Lin HH, Hilyard KL, Gordon S and McKnight AJ: Human epidermal growth factor (EGF) module-containing mucin-like hormone receptor 3 is a new member of the EGF-TM7 family that recognizes a ligand on human macrophages and activated neutrophils. J Biol Chem 276: 18863-18870, 2001.

45. Libby P: Inflammation in atherosclerosis. Nature 420: $868-874$ 2002.

46. Greaves DR and Gordon S: Immunity, atherosclerosis and cardiovascular disease. Trends Immunol 22: 180-181, 2001.

47. Libby P: Inflammation in atherosclerosis. Arterioscler Thromb Vasc Biol 32: 2045-2051, 2012.

48. Uhlén M, Fagerberg L, Hallström BM, Lindskog C, Oksvold P, Mardinoglu A, Sivertsson A., Kampf C, Sjöstedt E, Asplund A, et al: Proteomics. Tissue-based map of the human proteome. Science 347: 1260419, 2015.

49. Thul PJ, Åkesson L, Wiking M, Mahdessian D, Geladaki A Ait Blal H, Alm T, Asplund A, Björk L, Breckels LM, et al: A subcellular map of the human proteome. Science 356: eaal3321, 2017.

50. Wang C, Liu X, Liu Y, Zhang Q, Yao Z, Huang B, Zhang P, Li N and Cao X: Zinc finger protein 64 promotes Toll-like receptor-triggered proinflammatory and type I interferon production in macrophages by enhancing p65 subunit activation. J Biol Chem 288 : 24600-24608, 2013.
51. Somia NV, Schmitt MJ, Vetter DE, Van Antwerp D, Heinemann SF and Verma IM: LFG: An anti-apoptotic gene that provides protection from Fas-mediated cell death. Proc Natl Acad Sci USA 96: 12667-12672, 1999

52. Strasser A, Jost PJ and Nagata S: The many roles of FAS receptor signaling in the immune system. Immunity 30: 180-192, 2009.

53. Schütz C, Oelke M, Schneck JP, Mackensen A and Fleck M: Killer artificial antigen-presenting cells: The synthetic embodiment of a 'guided missile'. Immunotherapy 2: 539-550, 2010.

54. Lenardo MJ: Fas and the art of lymphocyte maintenance. J Exp Med 183: 721-724, 1996.

55. Hanada R, Leibbrandt A, Hanada T, Kitaoka S, Furuyashiki T, Fujihara H, Trichereau J, Paolino M, Qadri F, Plehm R, et al: Central control of fever and female body temperature by RANKL/RANK. Nature 462: 505-509, 2009.

56. Shimamura M, Nakagami $H$, Osako MK, Kurinami $H$, Koriyama H, Zhengda P, Tomioka H, Tenma A, Wakayama K and Morishita R: OPG/RANKL/RANK ax is is a critical inflammatory signaling system in ischemic brain in mice. Proc Natl Acad Sci USA 111: 8191-8196, 2014

(7)(9) This work is licensed under a Creative Commons Attribution-NonCommercial-NoDerivatives 4.0 International (CC BY-NC-ND 4.0) License. 